\title{
Preparation and Characterization of Baicalein-Loaded Nanoliposomes for Antitumor Therapy
}

\author{
Kun Li, ${ }^{1}$ Haizhao Zhang, ${ }^{1}$ Liming Gao, ${ }^{2}$ Yue Zhai, ${ }^{3}$ Ming Shi, ${ }^{1}$ Jian Li, ${ }^{1}$ \\ Chenlin Xiu, ${ }^{1}$ Junli Cao, ${ }^{2}$ Shaohui Cheng, ${ }^{2}$ Li Jiang, ${ }^{2}$ and ${\mathrm{Hao} \mathrm{Di}^{4}}^{4}$ \\ ${ }^{1}$ Department of Biological Engineering, College of Environment \& Chemical Engineering, Yanshan University, \\ No. 438 Hebei Street, Qinhuangdao 066004, China \\ ${ }^{2}$ The First Hospital of Qinhuangdao, No. 258, Wenhua Road, Haigang District, Qinhuangdao, Hebei 066000, China \\ ${ }^{3}$ The Hospital of Yanshan University, No. 438 Hebei Street, Qinhuangdao 066004, China \\ ${ }^{4}$ School of Finance, Nankai University, No. 38 Tongshuo Road, Tianjin 300350, China
}

Correspondence should be addressed to Kun Li; thebestlikun@163.com and Liming Gao; 17778889387@163.com

Received 1 April 2016; Revised 21 May 2016; Accepted 7 June 2016

Academic Editor: Mingqiang Li

Copyright (C) 2016 Kun Li et al. This is an open access article distributed under the Creative Commons Attribution License, which permits unrestricted use, distribution, and reproduction in any medium, provided the original work is properly cited.

Baicalein (BAI) is a major constituent of Scutellaria baicalensis Georgi. Previous studies showed that BAI had obvious effects on U14 cervical tumor-bearing mice model and HeLa cells. However, the use of BAI is inconvenient and troublesome, due to its low oral bioavailability. The aim of this study was to develop baicalein-loaded nanoliposomes (BAI-LP) to improve its bioavailability. In this study, BAI-LP was prepared by thin film hydration method. The average size, polydispersity index (PDI), zeta potential and encapsulation efficiency (EE) of the BAI-LP were $194.6 \pm 2.08 \mathrm{~nm}, 0.17 \pm 0.025,-30.73 \pm 0.41 \mathrm{mV}$, and $44.3 \pm 2.98 \%$, respectively. Drug storage stability study showed no significant changes in these values after 4 weeks of storing at $4^{\circ} \mathrm{C}$. Additionally, Sulforhodamine B (SRB) experimental results indicated that the BAI-LP could achieve better anti-tumor effects than free BAI. The results of the experiment demonstrated that BAI-LP had a better antitumor effect with a higher inhibition rate of $66.34 \pm 15.33 \%$ than free BAI with a inhibition rate of $41.89 \pm 10.50 \%$ by using U14 cervical tumor-bearing mice model. In conclusion, the study suggested that BAI-LP would serve as a potent delivery vehicle for BAI in future cancer therapy.

\section{Introduction}

Liposomes are microscopic vesicles which were originally found by British scholar Bangham et al. and composed of one or more lipid bilayers arranged in a concentric form enclosing an equal number of aqueous compartments [1]. Since the 1970s, researchers have begun to use it as a drug carrier for clinical diagnosis and treatment [2]. The main compounds of liposomes are phospholipid molecules and cholesterol. Liposomes can encapsulate both hydrophilic and lipophilic drugs. Basically, the lipophilic drugs have higher tendency to be entrapped in the lipid bilayers of liposomes. However, the hydrophilic drugs may be entrapped inside the aqueous cores of liposomes or located in the external water phase [3]. Liposomes can be divided into conventional, stealth, ligandtargeted, long-release, triggered-release, and long-circulating nanoliposomes based on different functions [4]. Liposomes have already been used in clinical treatments as carriers of anticancer drugs. In addition, an efficient enhanced permeability and retention (EPR) effect-based passive targeting of liposomes has been achieved. The EPR effect provides a great chance for passive targeting of liposomal anticancer agents into the tumor tissue, and this phenomenon has been widely observed in many solid tumors $[5,6]$. For example, Doxil is a kind of liposome used in clinical treatments which is used to entrap doxorubicin [7-9]. The Doxil formulation enhances the anticancer activity of doxorubicin by EPR effect $[10,11]$. At present, there are a lot of methods to prepare liposomes, such as thin film hydration method, ethanol injection method, freeze-drying method, ether injection method, and reverse phase evaporation method. [12]. In particular, thin film hydration method is most widely used and very suitable to encapsulate lipophilic drugs. 


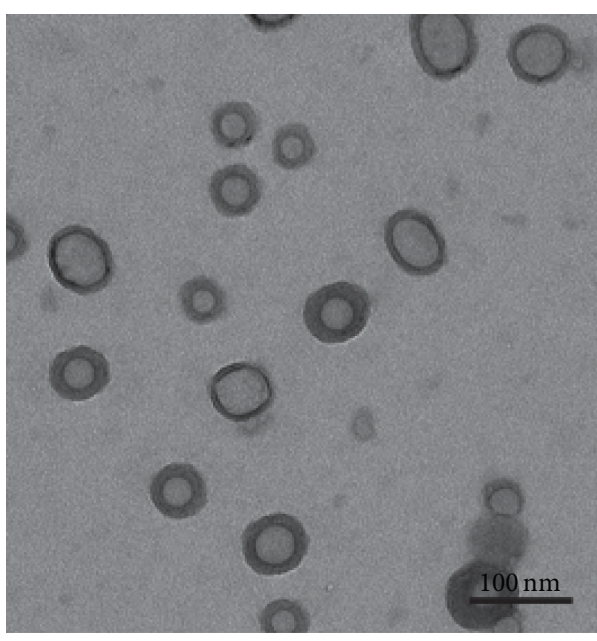

(a)

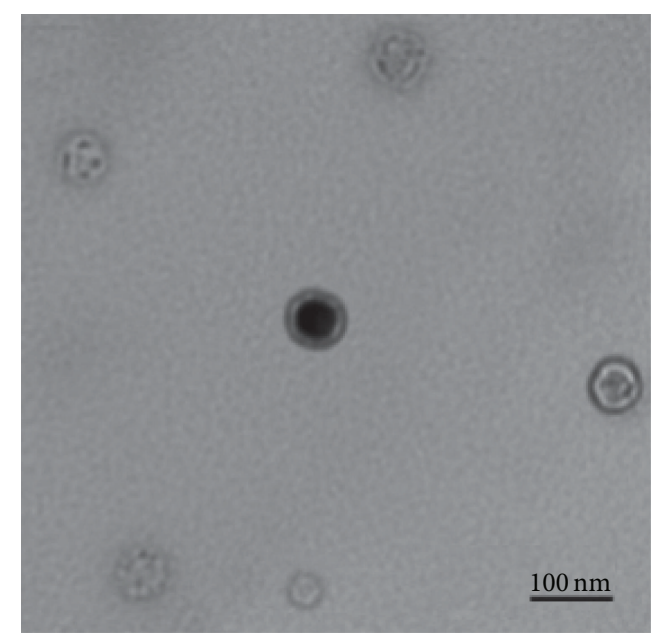

(b)

FIgURE 1: Transmission electronic microscopic images of liposomes. (a) LP; (b) BAI-LP.

BAI, a major constituent of Scutellaria baicalensis Georgi, has a variety of pharmacological effects such as antivirus, antibacterial, and anticancer [13]. According to previous studies, BAI is a very promising and attractive drug in treatment of bladder cancer [14]. Our research group has conducted years of researches on anticancer effects and mechanisms of BAI. The research results indicated that U14 cervical carcinoma cells and HeLa cells can be significantly inhibited by BAI. Toxicology tests showed that BAI could not lead mice to death even at the maximum tolerated dose. Though BAI has antitumor effects, the deficiencies such as low bioavailability and poor absorptions limited its clinical applications [15]. If encapsulated in liposomes, BAI will be easily absorbed by the human body due to the similarities between the bilayer of liposomes and biological membranes. Thus the bioavailability and the toxic side effects of BAI will be improved or reduced, respectively [16]. The object of this study was to improve bioavailability of BAI by preparing BAIloaded nanoliposomes (BAI-LP) using thin film hydration method, characterize BAI-LP in terms of morphology, average size, polydispersity index, zeta potential, EE, and stability, and explore the antitumor effects of BAI-LP.

\section{Materials and Methods}

2.1. Materials. Soybean phosphatidylcholine (SPC, AR) and cholesterol (CHOL, AR) were purchased from AVT Pharmaceutical Co., Ltd. Baicalein (BAI) (purity $\geq 98 \%$ ) was from XianTianbao Biotechnology Co., Ltd. Dichloromethane was obtained from Tianjin JingDongTianZheng Precision Chemical Reagent Factory. Methanol (AR) was purchased from Tianjin Kai Chemicals Co., Ltd. Methanol (LC) was from Tianjin Shield Company. Rotary evaporator was from Nobuo Ltd. Shanghai, China. Ultrasonic Cleaner KQ5200 was obtained from Kunshan Ultrasonic Instrument Co., Ltd. TEM (JEM-100CX/II) was produced by JEOL Ltd., Tokyo, Japan. TU-1950 UV-visible spectrophotometer was from Beijing Jingpin Seth Technology Co., Ltd. Zetasizer Nano-90 was purchased from the Malvern Instruments Ltd. U14 cervical carcinoma cells were purchased from the Beijing Cancer Hospital. Kunming mice were obtained from the Laboratory Animal Center of the Academy of Military Medical Sciences (China). All procedures involving animals were in accordance with institutional and governmental regulations about the use of experimental animals.

2.2. Preparation of LP and BAI-LP. A lipid phase was prepared by dissolving accurately weighed quantities of SPC, $\mathrm{CHOL}$, and $\mathrm{BAI}$ in $6 \mathrm{~mL}$ dichloromethane-methanol mixture $(2: 1, \mathrm{~V} / \mathrm{V})$, in a $25 \mathrm{~mL}$ recovery flask. The mixture was removed by rotary evaporation at $40^{\circ} \mathrm{C}$, to form a thin film of lipids on the wall of the eggplant-shaped bottle. Then the thin film was purged with nitrogen for 5 minutes. The lipids film was hydrated with $10 \mathrm{~mL}$ ultrapure water in the eggplant-shaped bottle at $60^{\circ} \mathrm{C}$, followed by sonication for $15-$ 30 minutes. $450 \mathrm{~nm}$ and $220 \mathrm{~nm}$ membrane filter were used to filter samples to obtain yellowish BAI-LP. Likewise, LP with Pan-blue light was obtained.

2.3. Characterization of LP and BAI-LP. The size, PDI, and zeta potential of LP and BAI-LP were determined using dynamic light scattering (DLS) techniques with a Zetasizer Nano-90 instrument at room temperature. The PDI was determined to check the level of homogeneity of particle size. The morphology of LP and BAI-LP was observed by TEM. A droplet of LP or BAI-LP was placed on a copper grid with carbon film for 10 minutes, followed by removal of the excess fluid with filter paper and then dried for $1 \mathrm{~h}$ at room temperature before examination on TEM.

2.4. Determination of Entrapment Efficiency and Drug Loading. The BAI was dissolved in a mixed solvent of ultrapure water and methanol $(1: 2, \mathrm{~V} / \mathrm{V})$ to obtain a concentration of $100 \mu \mathrm{g} / \mathrm{mL}$, and then the mixture was successively diluted to $50 \mu \mathrm{g} / \mathrm{mL}, 25 \mu \mathrm{g} / \mathrm{mL}, 12.5 \mu \mathrm{g} / \mathrm{mL}, 6.25 \mu \mathrm{g} / \mathrm{mL}$, and $3.125 \mu \mathrm{g} / \mathrm{mL}$ for standard curve. BAI detection was performed 


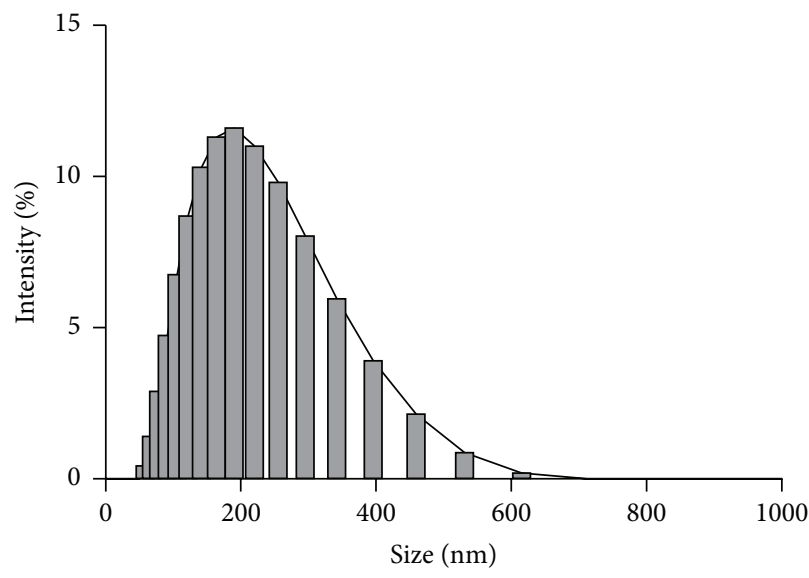

(a)

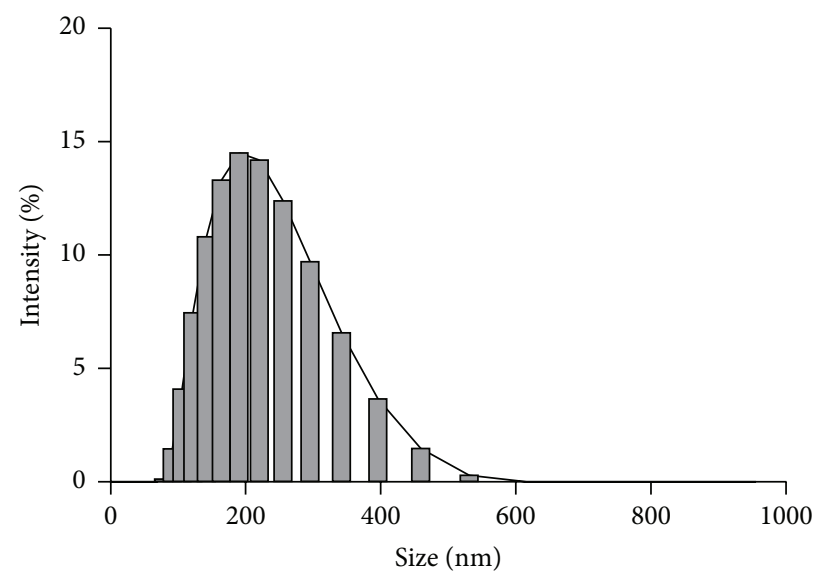

(b)

FIgURE 2: (a) Size distribution of LP; (b) size distribution of BAI-LP.

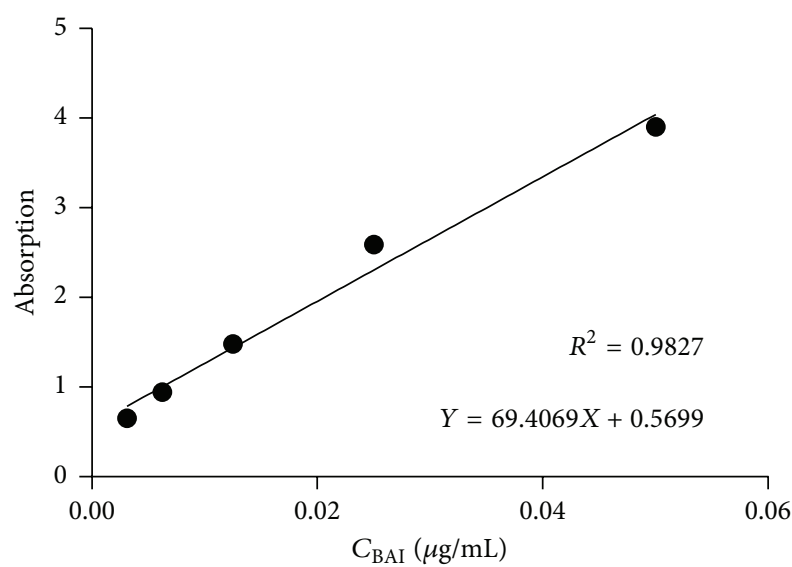

Figure 3: UV-visible absorption spectra of BAI solution.

using an UV-visible spectrophotometer at a wavelength of $275 \mathrm{~nm}$.

Separation of unentrapped drug from the liposomes was carried out by ultracentrifugation method. Appropriate amount of BAI-LP was placed in polycarbonate centrifuge tubes and centrifuged at $50,000 \times \mathrm{g}$ for 40 minutes at $4^{\circ} \mathrm{C}$. Subsequently, $1 \mathrm{~mL}$ of free BAI solution was transferred into a new centrifugal tube, and then $2 \mathrm{~mL}$ of methanol was added into this tube. The BAI detection was performed as described above. Its concentration was calculated according to the linear regression equation. $\mathrm{EE}$ and drug loading (DL) were calculated by using the following [17]:

$$
\begin{aligned}
& \mathrm{EE} \%=\frac{\left(W_{\text {total }}-W_{\text {free }}\right)}{W_{\text {total }}} \times 100 \%, \\
& \mathrm{DL} \%=\frac{\left(W_{\text {total }}-W_{\text {free }}\right)}{W_{\text {lipids }}} \times 100 \%,
\end{aligned}
$$

where $W_{\text {total }}$ is the analyzed weight of the drug in the dispersions and $W_{\text {free }}$ is the analyzed weight of free drug in

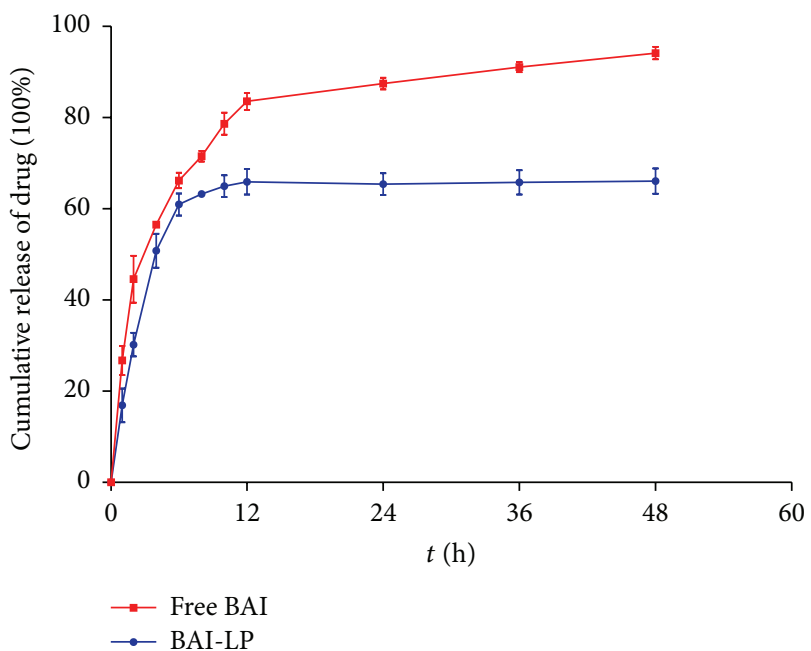

Figure 4: Release profiles in vitro of free BAI and BAI-LP. $(n=3)$.

the supernatant and $W_{\text {lipids }}$ is the total weight of the lipid content.

2.5. Drug Release In Vitro Study. The dialysis method was used to detect the in vitro release of BAI from liposomes. Briefly, $2 \mathrm{~mL}$ of free BAI and BAI-LP was added into the dialysis bags (molecular weight cutoff 3500 ), respectively. The dialysis bags were incubated in $40 \mathrm{~mL}$ PBS (pH 7.4) using a rotational speed of $100 \mathrm{rpm}$ at $37^{\circ} \mathrm{C}$. At predetermined time intervals, $2 \mathrm{~mL}$ of incubation medium was removed and the same volume of PBS ( $\mathrm{pH}$ 7.4) was added. In the end, BAI detection was performed using an UV-visible spectrophotometer at a wavelength of $275 \mathrm{~nm}$.

2.6. Stability of Liposomes. The LP and BAI-LP were evaluated for chemical and physical stability in the storage condition at $4^{\circ} \mathrm{C}$ for 4 weeks. The liposome samples were 


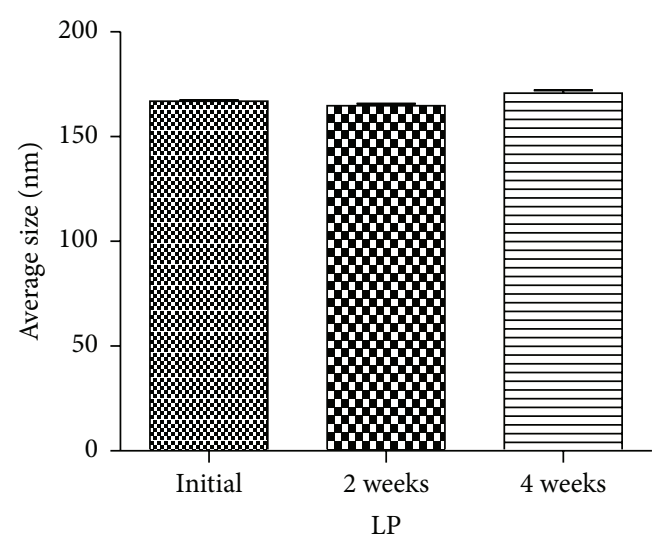

(a)

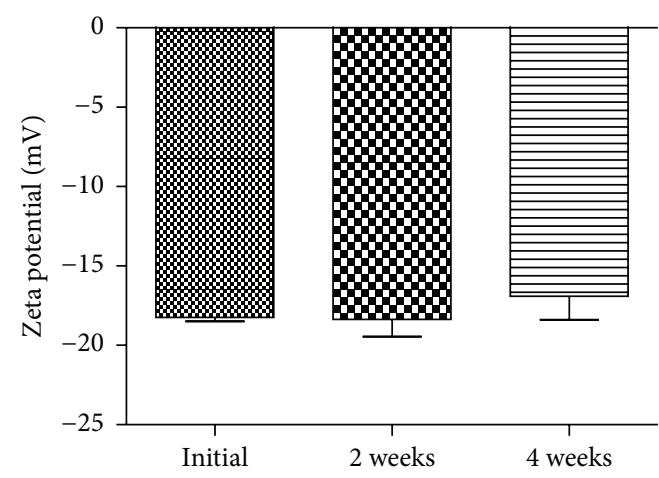

LP

(c)

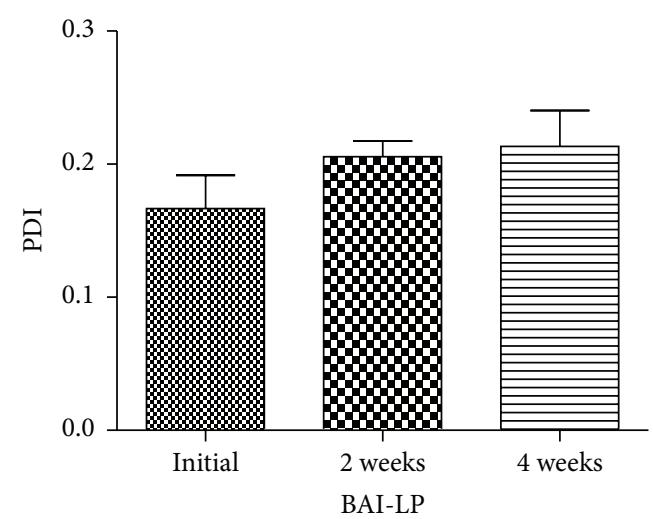

(e)

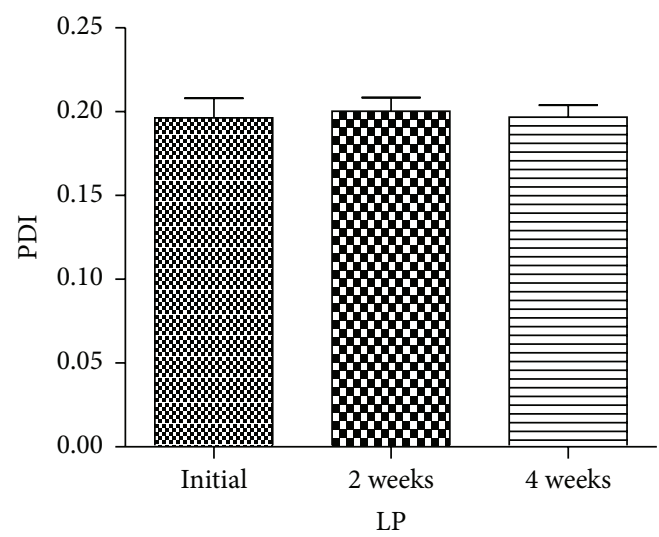

(b)

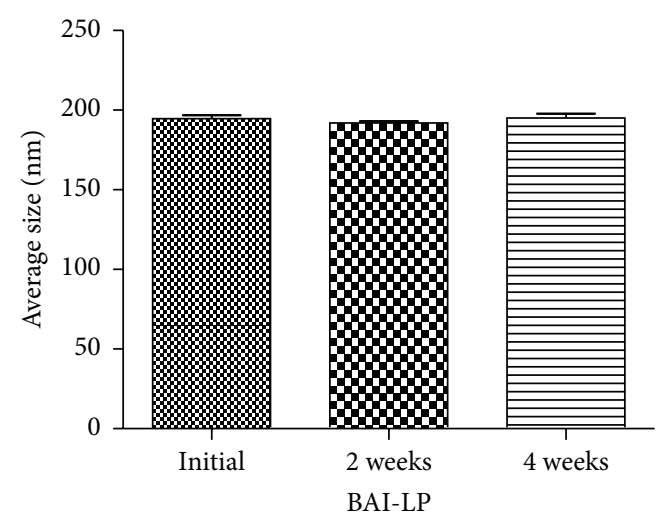

(d)

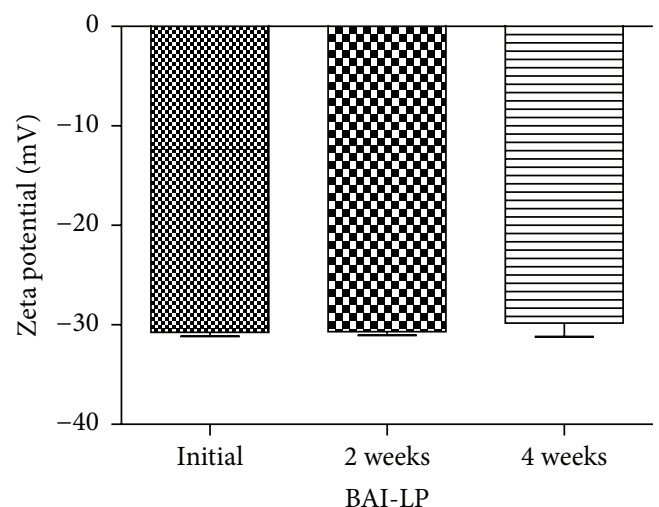

(f)

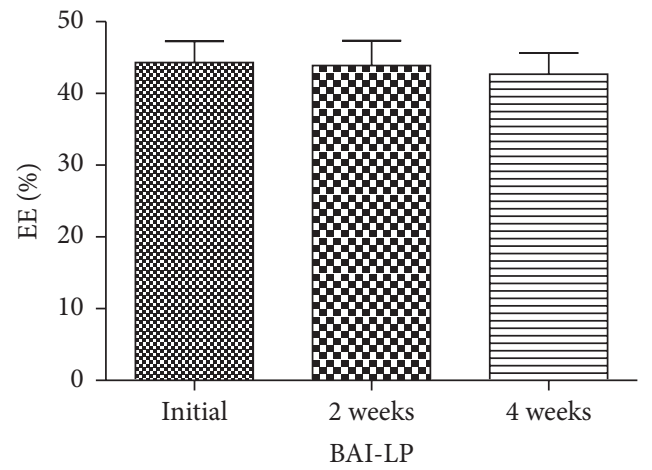

$(\mathrm{g})$

Figure 5: Stability of liposomes LP and BAI-LP at $4^{\circ} \mathrm{C}$ for 2 weeks and 4 weeks. (a) Average size of LP; (b) PDI of LP; (c) zeta potential of LP; (d) average size of BAI-LP; (e) PDI of BAI-LP; (f) zeta potential of BAI-LP; (g) EE of BAI-LP. 


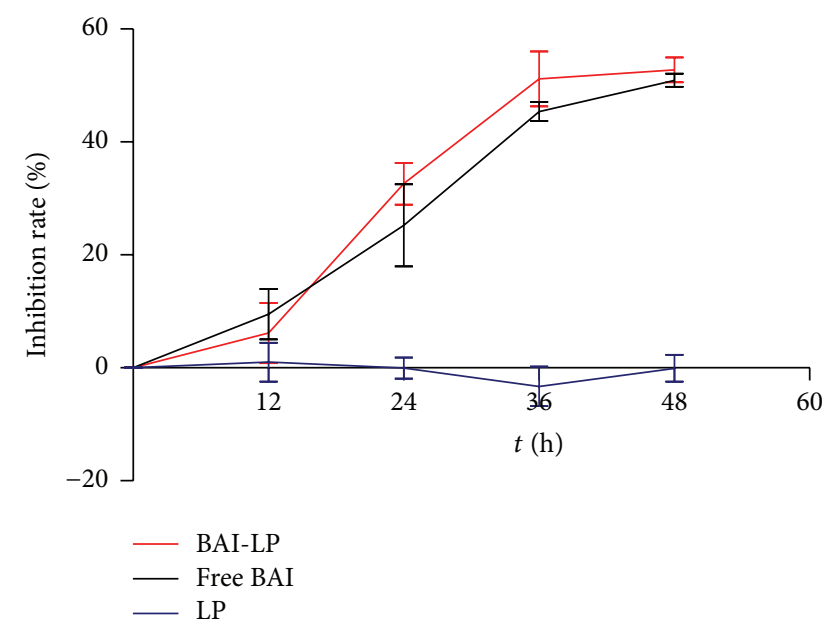

FIgUre 6: Anticancer effects of LP, free BAI, and BAI-LP. All values are expressed as mean $\pm \operatorname{SD}(n=6)$.

withdrawn at 2 weeks and 4 weeks, respectively, and characterized by the changes in average size, PDI, zeta potential, and EE [18].

2.7. Anti-Tumor Effects Assay In Vitro. In vitro, antitumor effects of LP, free BAI, and BAI-LP were evaluated by SRB colorimetric assay [19]. Briefly, HeLa cells were plated in 96well plates at a density of 10,000 cells/well and cultured for $24 \mathrm{~h}$. Then, the cells were treated with $50 \mu \mathrm{L}$ PBS, $50 \mu \mathrm{L} \mathrm{LP}$, $50 \mu \mathrm{L}$ free BAI $(100 \mu \mathrm{g} / \mathrm{mL})$, and $50 \mu \mathrm{L}$ BAI-LP $(100 \mu \mathrm{g} / \mathrm{mL})$ for $12 \mathrm{~h}, 24 \mathrm{~h}, 36 \mathrm{~h}$, and $48 \mathrm{~h}$, respectively. Afterward, the cells were fixed with cold trichloroacetic acid and dried at room temperature. The cells were then stained with $0.4 \%$
SRB for $30 \mathrm{~min}$ and $1 \%$ acetic acid was used to wash the excess dye. $10 \mathrm{mM}$ Tris base solution was used to dissolve the bound dye and then the absorbance was determined using a Thermo scientific multiskan FC microplate photometer at the wavelength of $540 \mathrm{~nm}(n=6)$.

\subsection{Determination of Tumor Inhibition Rate In Vivo}

2.8.1. U14 Mouse Cervical Carcinoma-Bearing Solid Tumor Model. To investigate the antitumor efficacy of free BAI and BAI-LP in vivo, we established the cervical carcinoma xenograft model through transplantation of the U14 cell line into female Kunming mice. Under the sterile condition, $0.3 \mathrm{~mL}$ of U14 cells $\left(1 \times 10^{6}\right.$ cells $\left./ \mathrm{mL}\right)$ was injected into the forelimb per mouse.

2.8.2. Groups and Treatment Scheme of Experimental Animals. The successfully vaccinated mice were divided into 4 groups, 8 for each group, and were named as follows: negative control group-intravenous administration of LP, $0.3 \mathrm{~mL}$; Positive control group-intragastric administration of $80 \mathrm{mg} / \mathrm{kg} \mathrm{BAI}$, $0.2 \mathrm{~mL}$ (our previous studies showed that this dose of BAI which was used for intragastric administration had obvious inhibitory effect on tumor growth); free BAI groupintravenous administration of $1 \mathrm{mg} / \mathrm{kg}$ free BAI, $0.3 \mathrm{~mL}$; BAILP group-intravenous administration of $1 \mathrm{mg} / \mathrm{kg}$ BAI-LP, $0.3 \mathrm{~mL}$; the mice were given the above-mentioned agents for 15 days.

2.8.3. Tumor Inhibition Rate. On the 16th day, the mice were sacrificed. Afterward, the tumor tissues taken from the mice were weighed to detect inhibition rates. Inhibitory rates were calculated according to the following:

$$
\text { Inhibition rate }=\frac{(\text { negative group tumor mass }- \text { experimental group tumor mass })}{\text { negative group tumor mass }} \times 100 \%
$$

2.9. Statistics. Data analyses were performed using Graphpad Prism 5 software and the results were shown as mean \pm standard deviation. One-way ANOVA was used to evaluate the differences between groups, and $p<0.05$ was considered as statistically significant.

\section{Results and Discussion}

3.1. Physical and Chemical Properties of LP and BAI-LP. LP and BAI-LP prepared by the thin film hydration method were found to be a mildly translucent liposomal suspension. We first explored the optimal conditions for preparation of LP and BAI-LP in terms of components of BAI, cholesterol, and SPC; we found that the appropriate ratio of cholesterol to SPC $(\mathrm{w} / \mathrm{w})$ was $1: 4$ and that the optimal ratio of drug to SPC (w/w) was $1: 8$. Likewise, the optimal temperature for film-forming and hydration was $40^{\circ} \mathrm{C}$ and $60^{\circ} \mathrm{C}$, respectively. Based on the above conditions, we successfully prepared LP, Pan-blue light suspension, and BAI-LP, yellowish suspension.
3.1.1. The Morphology of LP and BAI-LP. The images in Figure 1 showed that the liposome nanoparticles were clearly separated from each other. The shape of the LP and BAILP was spherical with smooth surface. Furthermore, the obvious lipid bilayer of the LP and BAI-LP can be seen clearly. However, there were obvious changes between LP and BAILP in the morphology. Figure 1(a) showed that the inside of LP is blank. Figure 1(b) showed the inner black dots and the outer darker ring in BAI-LP corresponding to BAI and the phospholipids' vesicles, respectively [20].

3.1.2. The Size, PDI, and Zeta Potential of LP and BAI-LP. The results of DLS analysis were presented in Figure 2. The average size of LP was $166.9 \pm 0.43 \mathrm{~nm}$ (PDI $0.19 \pm 0.012$ ) with no obvious aggregation. Similarly, the average size of BAI-LP was $194.6 \pm 2.08 \mathrm{~nm}$ (PDI $0.17 \pm 0.025)$. Notably, the average size and the size distribution of BAI-LP are much smaller than those of BAI-LP prepared by diethyl ether injection 


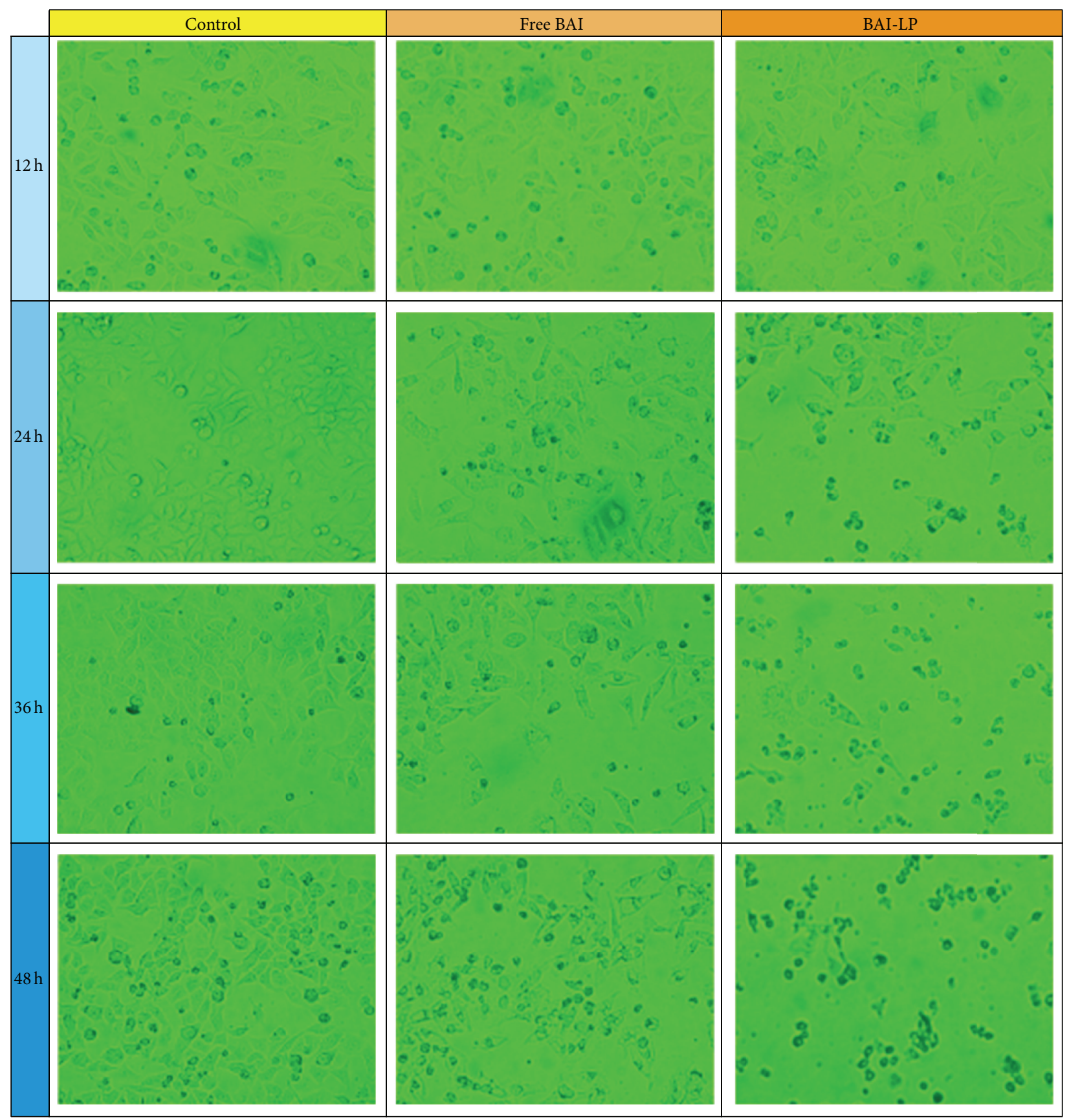

FIgURE 7: Images of HeLa cells incubation with PBS (control), free BAI, and BAI-LP.

method [14]. The PDI was determined to examine the level of uniformity of particle size. Hence, the above results indicate that the particle size of LP and BAI-LP was uniform, which may prevent the agglomerations.

Zeta potential measurements showed that the surface charges of LP and BAI-LP were $-18.23 \pm 0.25 \mathrm{mV}$ and $-30.73 \pm$ $0.41 \mathrm{mV}(n=3)$, respectively. A large zeta potential could prevent the agglomerations between liposome particles and thus maintained their nanoscaled morphology.
3.1.3. Drug Entrapment Efficiency and Drug Loading of BAI$L P$. Known quantities of BAI were added into ultrapure water and methanol mixed solvent $(1: 2, \mathrm{~V} / \mathrm{V})$, and then the resultant samples were analyzed by UV-visible spectrophotometer. The absorption measurement showed that BAI concentration was linear over the range of $3.125-50 \mu \mathrm{g} / \mathrm{mL}$ with a regression equation: $Y=69.4069 X+0.5699$. The correlation coefficient was 0.9827 . The UV-visible absorption spectra of BAI solution are shown in Figure 3. 

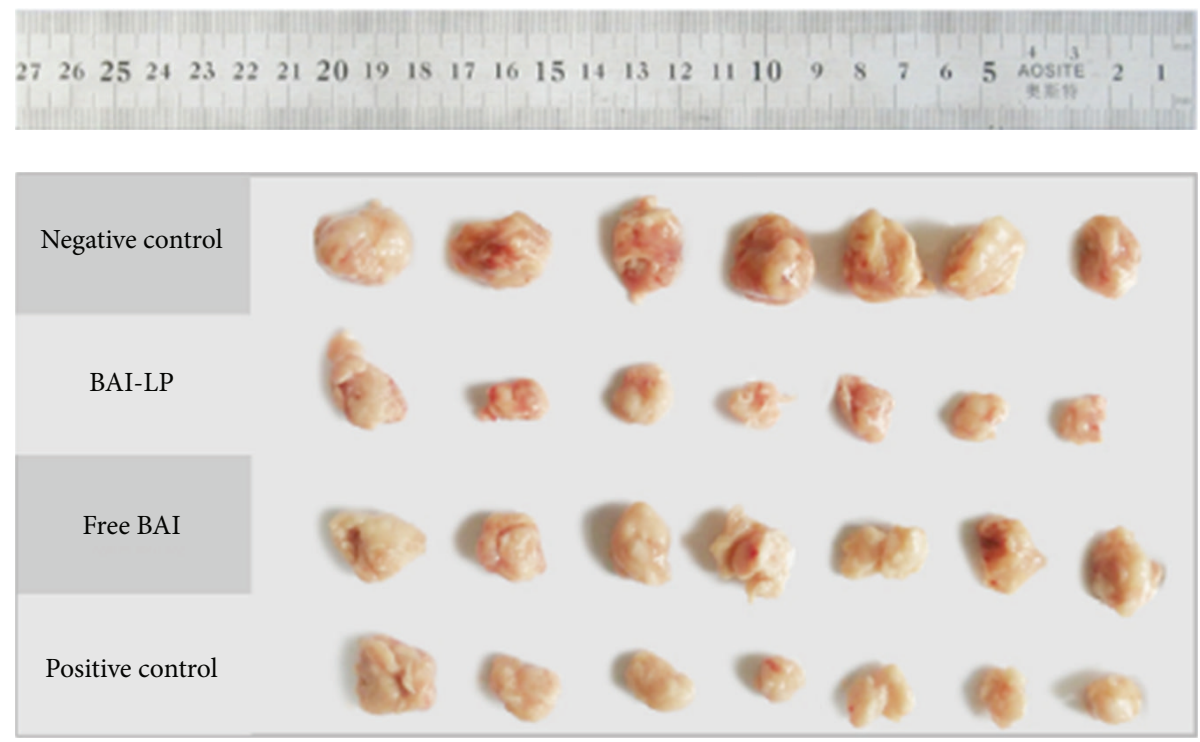

FIGURE 8: Photographs of the representative tumor tissues isolated from tumor-bearing mice after 15-day treatments.

The concentration of free BAI was calculated according to the linear regression equation. The final entrapment efficiency of BAI-LP was $44.3 \pm 2.98 \%$, which was similar to previous report [14]. Compared with published data on liposomal drug delivery systems of herbal extracts or compounds from herbal origin, the entrapment efficiency was satisfactory. The drug loading of BAI-LP was $4.41 \pm 0.02 \%$.

3.1.4. Drug Release In Vitro Study. The release behaviors of BAI from the liposomes in vitro were shown in Figure 4 . The release profiles of BAI-LP contained two stages including an initial stage of relatively large burst effect and a slower release stage. As shown in Figure 4, the free BAI exhibited the fastest release rate and more than $92 \%$ BAI was released within $48 \mathrm{~h}$. However, the release rate of BAI-LP was $65 \%$ within $48 \mathrm{~h}$ which was less than that of free BAI. Thus a drug depot effect could be achieved by the liposomes. The BAI-LP showed a more stable state and slow drug release in $48 \mathrm{~h}$.

3.1.5. Stability of Liposomes. Stability of LP and BAI-LP was important from the nanomedicine point of view. The agglomerations between liposome particles and the leakage of the encapsulated drug may occur, when liposomes were kept for a long time. In this study, no remarkable agglomerations or leakage for BAI-LP was observed in the storage condition at $4^{\circ} \mathrm{C}$ after 2 and 4 weeks. No obvious changes in average size, PDI, zeta potential, and EE for LP and BAI-LP were observed. In particular, the average size of LP was about $170 \mathrm{~nm}$ after 2 and 4 weeks. Similarly, the average size of BAI-LP was about $190 \mathrm{~nm}$ and $96 \%$ of drug was entrapped (Figure 5).

3.2. Antitumor Effects Assay In Vitro. The antitumor effects of LP, free BAI, and BAI-LP in vitro were investigated. SRB assay revealed the time-dependent antitumor effects of LP, free BAI, and BAI-LP. As shown in Figure 6, the HeLa cells treated with LP exhibited $1.02 \pm 3.43 \%,-0.02 \pm 1.85 \%,-3.27 \pm$
$3.48 \%$, and $-0.08 \pm 2.37 \%$ cell inhibitory rates at $12 \mathrm{~h}, 24 \mathrm{~h}$, $36 \mathrm{~h}$, and $48 \mathrm{~h}$, respectively. Hence, the LP had no effect on the growth of cancer cells. The HeLa cells treated with free BAI $\left(66 \mu \mathrm{g} \cdot \mathrm{mL}^{-1}\right)$ exhibited $9.52 \pm 4.46 \%, 25.24 \pm 7.24 \%$, $45.38 \pm 1.71 \%$, and $50.90 \pm 1.17 \%$ cell inhibitory rates at $12 \mathrm{~h}$, $24 \mathrm{~h}, 36 \mathrm{~h}$, and $48 \mathrm{~h}$, respectively. The HeLa cells treated with BAI-LP $\left(66 \mu \mathrm{g} \cdot \mathrm{mL}^{-1}\right)$ exhibited $6.15 \pm 5.34 \%$, $32.61 \pm 3.71 \%$, $51.19 \pm 4.85 \%$, and $52.77 \pm 2.19 \%$ cell inhibitory rates at $12 \mathrm{~h}, 24 \mathrm{~h}, 36 \mathrm{~h}$, and $48 \mathrm{~h}$, respectively. Notably, compared to the free BAI, BAI-LP enhanced tumoricidal effect at $24 \mathrm{~h}$, $36 \mathrm{~h}$, and $48 \mathrm{~h}$. The anticancer effect of BAI was improved by entrapping BAI into liposomes. However, the inhibitory rates of BAI-LP were lower than free BAI at $12 \mathrm{~h}$. This may be due to the action of liposome, which has the slow-released drug effect. As shown in Figure 7, more dead cells were observed in the treatments of free BAI and BAI-LP, which conformed that BAI can inhibit the tumor cells growing. The antitumor effect of BAI-LP was most remarkable. The high inhibition rates of BAI-LP are probably due to the reason that the lipid bilayers of liposomes can improve drug permissive into the cell membrane and eventually enhance the penetration of drugs.

3.3. Tumor Inhibition Rate. On the third day after inoculation of U14 cervical cancer cells, tumors grew well at mice armpits, indicating that the vaccination was successful and the success rate was $100 \%$. Figure 8 showed the photographs of the representative tumor tissues isolated from tumor-bearing mice after 15-day treatments. The tumors inhibition rate of each mouse group was calculated according to (2). The results are shown in Figure 9 and Table 1. The BAI-LP group had the similar inhibitory effect on tumor growth with the positive control group. Obviously, BAI-LP improved the inhibition rate compared with free BAI group $(p<0.01)$. This may be related to the fact that BAI-LP showed a more stable state and slow drug release. Hence, BAI-LP could release 
TABLE 1: The tumors inhibition rate of each mouse group $(n=8)$.

\begin{tabular}{lccc}
\hline Group & Positive control & Free BAI & BAI-LP \\
\hline Inhibition rate (\%) & $60.90 \pm 10.05$ & $41.89 \pm 10.50$ & $66.34 \pm 15.33$ \\
\hline
\end{tabular}

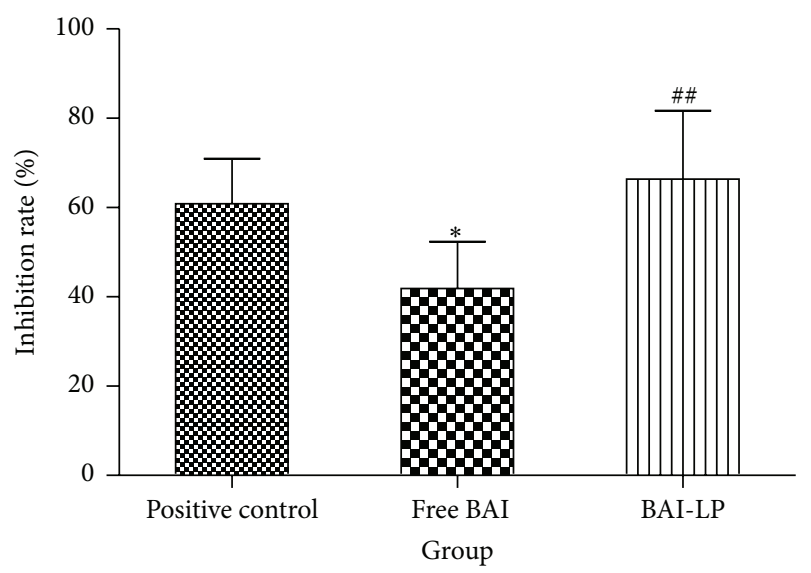

FIGURE 9: Histogram of inhibition rate of mice in each group. Compared with positive group, ${ }^{*} p<0.05$. Compared with free BAI group, ${ }^{\#} p<0.01(n=8)$.

drug slower than free BAI to achieve a better therapeutic effect. Moreover, liposomes not only improve the solubility of BAI but also enhance the absorption due to the similarities between the bilayer of liposomes and biological membranes. Furthermore, the pores in tumor endothelium were larger than $100 \mathrm{~nm}$, while the spaces in endothelial junctions of normal vessels were $5 \mathrm{~nm}$ to $10 \mathrm{~nm}$ in size. Hence, the BAILP with $194.6 \pm 2.08 \mathrm{~nm}$ could reduce rapid clearance and prolong time of blood cycle to reach tumor tissues.

\section{Conclusion}

As is demonstrated above, the thin film hydration was a useful method to prepare highly dispersed nanoscaled BAI-loaded nanoliposomes, which had a smaller particle size of distinct spherical vesicles with narrow distribution and a similar EE of BAI compared with diethyl ether injection method in previous studies. The uniform particle size and the large zeta potential of LP and BAI-LP prevent the agglomerations between liposome particles and stabilize for at least 4 weeks. The tumor inhibition rate of BAI-LP was much higher than BAI's in vitro and in vivo. Hence, the study suggests that BAILP may serve as a promising approach for cancer therapy.

\section{Competing Interests}

The authors declare that they have no direct financial relation with the commercial identities mentioned in this paper that might lead to a conflict of interests for any of the authors.

\section{Acknowledgments}

This study was supported by "Qinhuangdao Science and Technology Research and Development Plan," China, no. 201501B034, no. 201401A105, no. 201501B051, and no. 201502A193, and Special Research Found for the Doctoral Program of Higher Education, no. 20121333120017.

\section{References}

[1] A. D. Bangham, M. M. Standish, and J. C. Watkins, "Diffusion of univalent ions across the lamellae of swollen phospholipids," Journal of Molecular Biology, vol. 13, no. 1, pp. 238-252, 1965.

[2] J. H. Kim and M. W. Kim, "In-situ observation of the insideto-outside molecular transport of a liposome," The Journal of Physical Chemistry B, vol. 112, no. 49, pp. 15673-15677, 2008.

[3] Z. Fang and B. Bhandari, "Encapsulation of polyphenols-a review," Trends in Food Science and Technology, vol. 21, no. 10, pp. 510-523, 2010.

[4] Y. P. Patil and S. Jadhav, "Novel methods for liposome preparation," Chemistry and Physics of Lipids, vol. 177, pp. 8-18, 2014.

[5] P. G. Schmidt, J. P. Adler-Moore, E. A. Forssen et al., "Unilamellar liposomes for anticancer and antifungal therapy," in Medical Applications of Liposomes, pp. 703-731, Elsevier, New York, NY, USA, 1998.

[6] R. Solomon and A. A. Gabizon, "Clinical pharmacology of liposomal anthracyclines: focus on pegylated liposomal doxorubicin," Clinical Lymphoma and Myeloma, vol. 8, no. 1, pp. 21-32, 2008.

[7] Y.-M. Ning, K. He, R. Dagher et al., "Liposomal doxorubicin in combination with bortezomib for relapsed or refractory multiple myeloma," Oncology, vol. 21, no. 12, pp. 1503-1516, 2007.

[8] L. Bracci, G. Schiavoni, A. Sistigu, and F. Belardelli, "Immunebased mechanisms of cytotoxic chemotherapy: implications for the design of novel and rationale-based combined treatments against cancer," Cell Death and Differentiation, vol. 21, no. 1, pp. 15-25, 2014.

[9] A. Gabizon, H. Shmeeda, and Y. Barenholz, "Pharmacokinetics of pegylated liposomal doxorubicin: review of animal and human studies," Clinical Pharmacokinetics, vol. 42, no. 5, pp. 419-436, 2003.

[10] J. Fang, H. Nakamura, and H. Maeda, “The EPR effect: unique features of tumor blood vessels for drug delivery, factors involved, and limitations and augmentation of the effect," Advanced Drug Delivery Reviews, vol. 63, no. 3, pp. 136-151, 2011.

[11] H. Maeda, "Macromolecular therapeutics in cancer treatment: the EPR effect and beyond," Journal of Controlled Release, vol. 164, no. 2, pp. 138-144, 2012.

[12] V. P. Torchilin, "Recent advances with liposomes as pharmaceutical carriers," Nature Reviews Drug Discovery, vol. 4, no. 2, pp. 145-160, 2005.

[13] S. Li and Y. Chen, "Advances in flavonoids mechanism of action of lung cancer," Rational Drug Use, vol. 4, pp. 172-174, 2014.

[14] J. Liang, W. Wu, Q. Liu, and S. Chen, "Long-circulating nanoliposomes (LCNs) sustained delivery of baicalein (BAI) with desired oral bioavailability in vivo," Drug Delivery, vol. 20, no. 8, pp. 319-323, 2013.

[15] S. Ikemoto, K. Sugimura, N. Yoshida et al., "Antitumor effects of Scutellariae radix and its components baicalein, baicalin, and wogonin on bladder cancer cell lines," Urology, vol. 55, no. 6, pp. 951-955, 2000

[16] F. M. Menger, J. S. Keiper, and S. J. Lee, "Adhesion of giant liposomes as observed by light microscopy," Langmuir, vol. 13, no. 17, pp. 4614-4620, 1997. 
[17] W. Sun, N. Zhang, A. Li, W. Zou, and W. Xu, "Preparation and evaluation of $\mathrm{N}_{3}$-O-toluyl-fluorouracil-loaded liposomes," International Journal of Pharmaceutics, vol. 353, no. 1-2, pp. 243250, 2008.

[18] Y. Wei, Z. Xue, Y. Ye, Y. Huang, and L. Zhao, "Paclitaxel targeting to lungs by way of liposomes prepared by the effervescent dispersion technique," Archives of Pharmacal Research, vol. 37, no. 6, pp. 728-737, 2014.

[19] V. Vichai and K. Kirtikara, "Sulforhodamine B colorimetric assay for cytotoxicity screening," Nature Protocols, vol. 1, no. 3, pp. 1112-1116, 2006.

[20] S. Xu, B. Zhao, and D. He, "Synthesis of highly dispersed nanoscaled $\mathrm{CoQ}_{10}$ liposome by supercritical fluid," Materials Letters, vol. 142, pp. 283-286, 2015. 

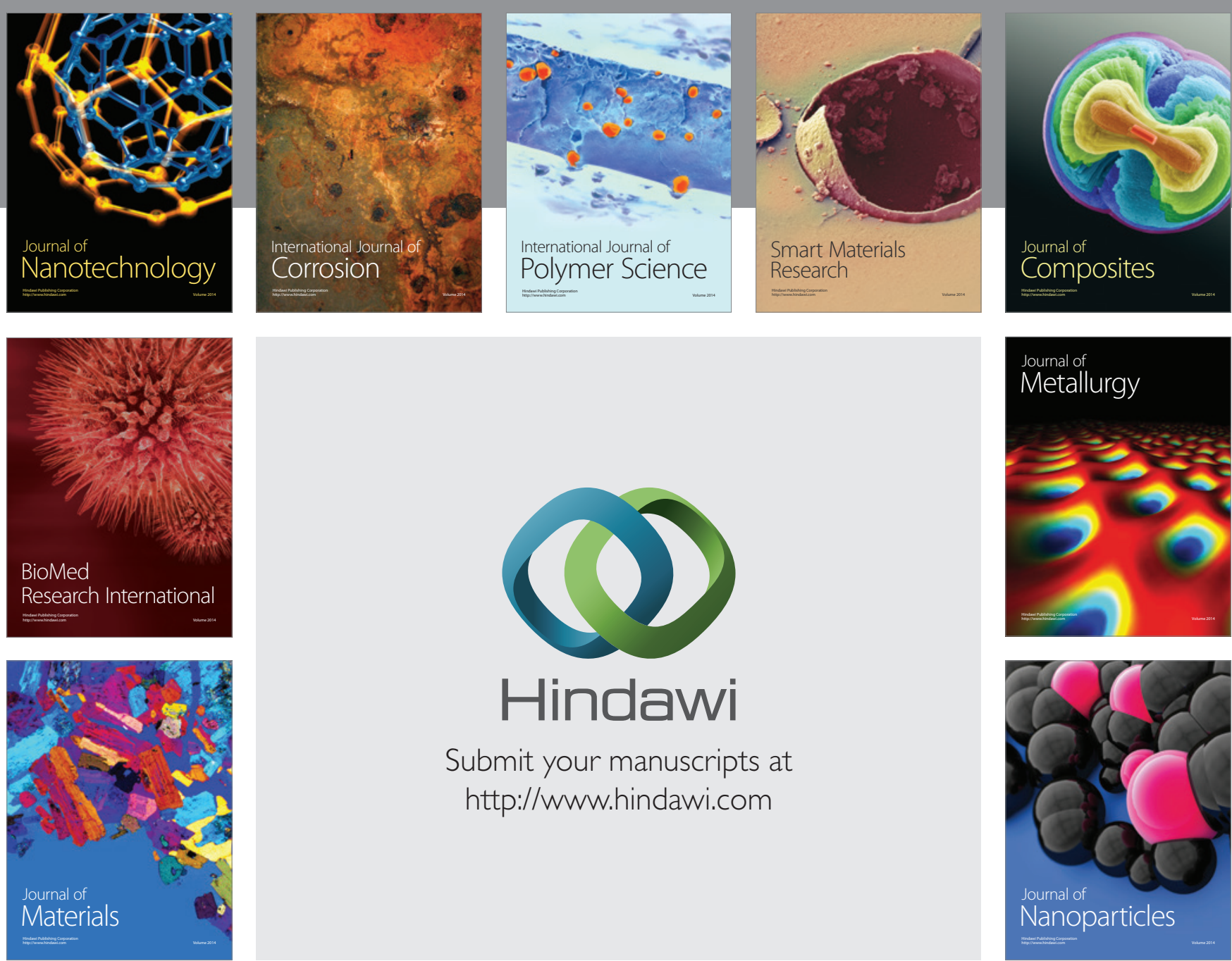

\section{Hindawi}

Submit your manuscripts at

http://www.hindawi.com

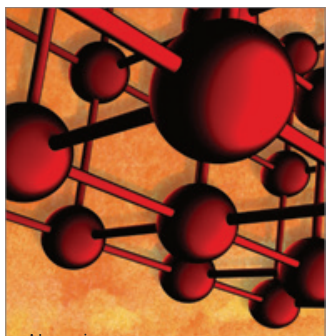

Materials Science and Engineering
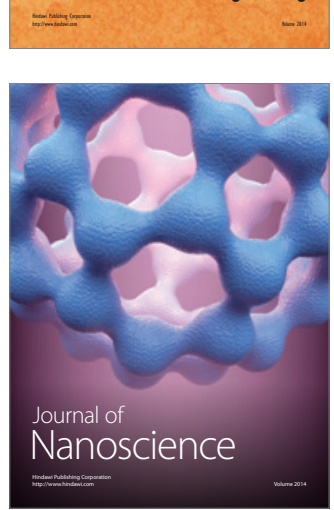
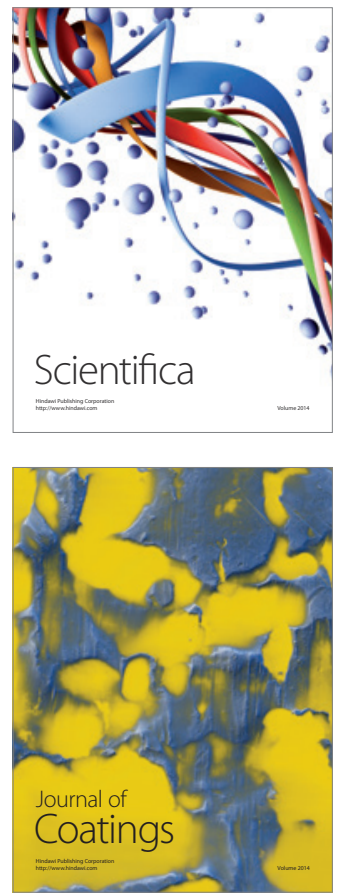
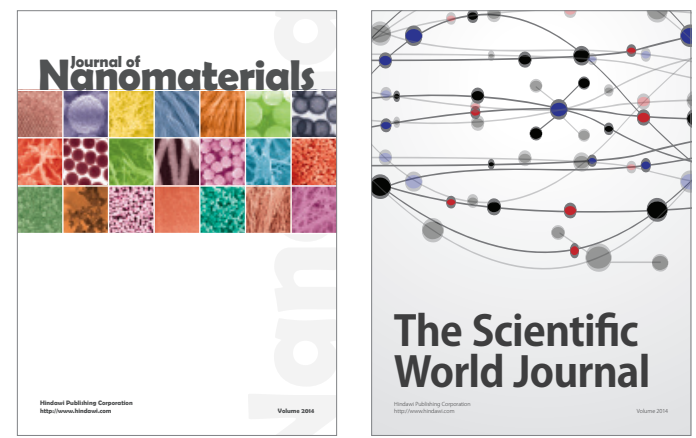

The Scientific World Journal
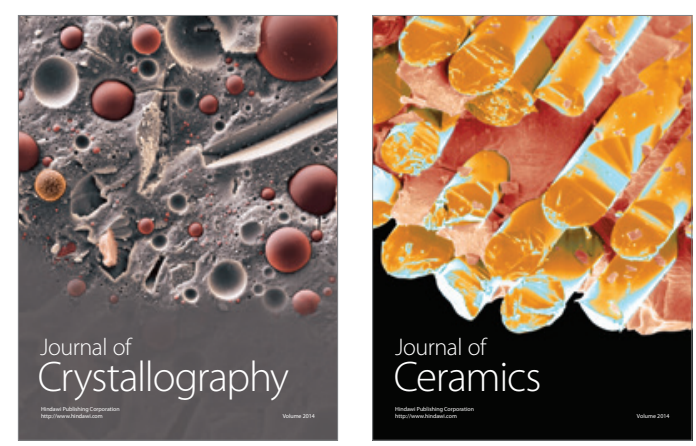
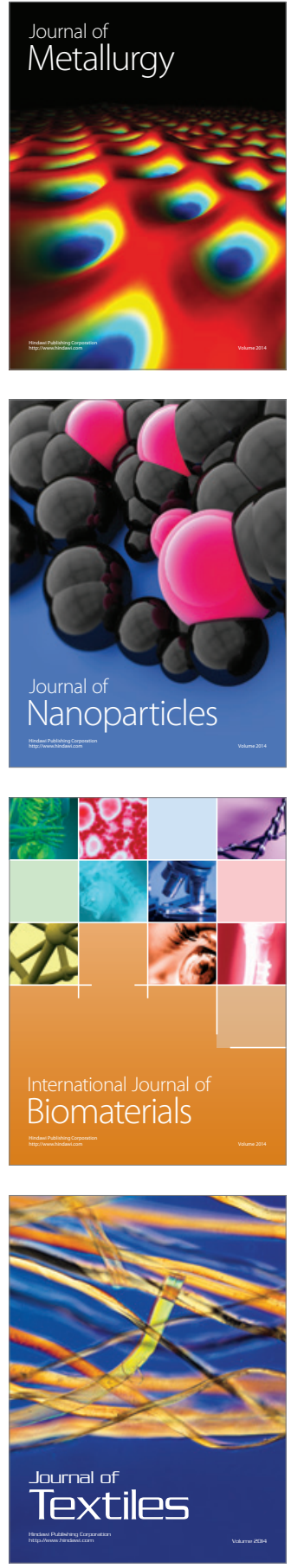